\title{
An Optimal Control Experiment for an SEIRS Epidemiological Model
}

\author{
Tanner Snyder*, Ryan Nierman \\ Department of Mathematics and Computer Science, Madonna University, Livonia, MI \\ https://doi.org/10.33697/ajur.2021.047 \\ Student: tsnyder213@my.madonna.edu* \\ Mentor:rnierman@madonna.edu
}

\begin{abstract}
This work studies an optimal control model for a discrete-time Susceptible/Exposed/Infective/Removed/Susceptible (SEIRS) deterministic epidemiological model with a finite time horizon and changing population. The model presented converts a continuous SEIRS model that would typically be solved using differential equations into a discrete model that can be solved using dynamic programming. The discrete approach more closely resembles real life situations, as the number of individuals in a population, the rate of vaccination to be applied, and the time steps are all discrete values. The model utilizes a previously developed algorithm and applies it to the presented SEIRS model. To demonstrate the applicability of the algorithm a series of numerical results are presented for various parameter values.
\end{abstract}

\section{KEYWORDS}

Control; Cost; Discrete; Disease; Epidemiology; Minimization; Modeling; Optimality; SEIRS; Vaccination

\section{INTRODUCTION}

Epidemiological modeling relies on the usage of numerous epidemiological classes. The most common are described by $M, S, E, I$, and $R$. $M$ represents the class of individuals who have passive immunity, $S$ represents the susceptible class (individuals who are capable of becoming infected), $E$ represents the exposed class (individuals who are infected, but not yet infectious), $I$ represents the infected class (those who are infectious and capable of spreading the disease to a member of the susceptible class), and $R$ represents the recovered or removed class. This class may consist of those with either permanent infectious-acquired immunity, temporary immunity, or those who have died/emigrated, and therefore, have been removed from the population. Some of the most common epidemiological models include: MSEIR, MSEIRS, SEIR, SEIRS, SIR, SIRS, SEI, SEIS, SI, and SIS. 2, 4, 8, 15, 16 Figure 1 shows the general transfer diagram for a MSEIR model. ${ }^{10}$

The research presented explores a Susceptible/Exposed/Infective/Removed/Susceptible (SEIRS) model. Typically, epidemiological models are represented by one or more ordinary or partial differential equations, which are then used to model the spread of the epidemic or solve questions of optimality. ${ }^{9,} 17$ In the case of the research presented, the continuous model is converted to a discrete model in which a nonlinear programming method for finding the optimal control sequence is applied for the discrete-time deterministic SEIRS epidemic. The algorithm will seek to find the minimum cost necessary to control an epidemic under a finite time horizon and discrete state space. The numerical results and corresponding graphs are generated using a python script. ${ }^{\mathrm{A}}$

\footnotetext{
A The python script can be found on GitHub at https://github.com/tsnyder213/SEIRS-Optimality
} 


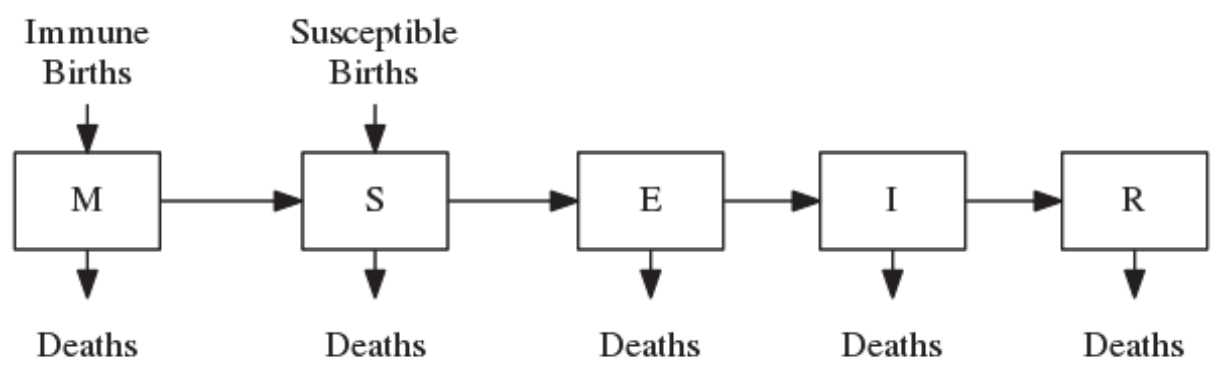

Figure 1. A General Transfer Diagram for the MSEIR Model.

\section{AN SEIRS EPIDEMIC OPTIMAL CONTROL PROBLEM}

A typical Susceptible/Exposed/Infective/Removed/Susceptible epidemiological model is presented using a system of differential equations. The total population $N$ is divided into multiple subpopulations/classes, namely, $S, E, I$, and $R$, where $S$ represents the susceptibles, $E$ represents the exposed class (or those that are infected, but not yet infectious), $I$ represents the infectives, and $R$ represents those that have been removed from the population due to death as a result of the disease. A basic SEIRS model assumes that the total population, $N$, does not allow for natural births/deaths nor immigration/emigration, therefore individuals are only able to move between subpopulations. Having said that, the model utilized in the research explores a more advanced model that allows for a changing population. Additionally, the model assumes that it is possible for an individual to become infectious, recover from the disease, and reenter the susceptible class, which is why the model presented represents an SEIRS model, rather than an SEIR one.

The following systems of differential equations represents a common SEIRS model without natural births/deaths or immigration/emigration included. ${ }^{1,7,13}$

$$
\begin{gathered}
\frac{d S}{d t}=-\frac{\beta I S}{N}+\tau I \\
\frac{d E}{d t}=\frac{\beta I S}{N}-\mu E \\
\frac{d I}{d t}=\mu E-\gamma I-\tau I \\
\frac{d R}{d t}=\gamma I .
\end{gathered}
$$

Equation 1.

Equation 2.

Equation 3.

Equation 4.

In this model, $\beta \in[0,1]$ represents the probability of infection when an infective comes into contact with a susceptible, $\tau \in[0,1]$ represents the rate of recovery, $\mu \in[0,1]$ represents the rate of people leaving the exposed class and entering the class of infectives, and $\gamma \in[0,1]$ represents the rate of those removed from $I$ as a result of dying from the disease. ${ }^{5,8,9,12,14-16}$ The term

$$
\frac{\beta I S}{N}
$$

Equation 5.

is referred to as the interaction term because it represents the interaction between an infective and a susceptible and the effect that $\beta$ has on transmission during that interaction. ${ }^{1,10,13,15,16}$

To allow the total population $N$ to change due to natural births/deaths and/or immigration/emigration, the previous system of differential equations outlined in Equations 1-4 becomes:

$$
\begin{array}{ll}
\frac{d S}{d t}=\phi N-\frac{\beta I S}{N}+\tau I-\delta_{1} S & \text { Equation } 6 . \\
\frac{d E}{d t}=\frac{\beta I S}{N}-\mu E-\delta_{2} E & \text { Equation 7. }
\end{array}
$$




$$
\begin{array}{ll}
\frac{d I}{d t}=\mu E-\gamma I-\tau I-\delta_{3} I & \text { Equation } 8 . \\
\frac{d R}{d t}=\gamma I+\delta_{1} S+\delta_{2} E+\delta_{3} I, & \text { Equation 9. }
\end{array}
$$

where $\phi$ a rate of population growth due to natural births or immigration. This term can be referred to as the recruitment rate of the susceptibles. It is assumed that all natural births and immigration result in new susceptibles, therefore, no one can enter the population and immediately be entered into the class of exposed or infectious individuals. Furthermore, $\delta_{1}, \delta_{2}$, and $\delta_{3}$ represent the rates of natural deaths or emigration unrelated to the disease. It is assumed that $\phi \in[0,1], \delta_{1} \in[0,1], \delta_{2} \in[0,1]$, and $\delta_{3} \in[0,1] .^{7}$

Having established the continuous representation for an SEIRS model, it is now necessary to convert the model into a discrete model, which can be used to establish an optimal treatment strategy to minimize the cost of controlling the spread of the epidemic. By relying on the use of discrete mathematics, the model will be able to overcome issues with convergence and ill-behaved functions. Discrete modeling is more realistic than continuous models since it relies on discrete time steps and individuals within a population. ${ }^{15,16}$ Therefore, instead of using ordinary differential equations to express changes in epidemiological classes over time (i.e., the rates at which individuals are moving between subpopulations), $S, E, I$ and $R$ are going to be based solely on previous discrete time steps. The control setting is an epidemic process with a finite time horizon and discrete state space. Let the epidemic evolve over integral decision times $\{T \in \mathbb{N} \mid 0 \leq T<\infty\}$. At the start of each time interval $(t, t+1)$, a decision, $u_{t}$, specifying the rate of vaccination (control) to apply must be made. Hence, $u_{t}$ is an element of a vector $u$ of decisions of length $T$. The number of possible control rates is based on the number of partitions/partial rates of vaccination (control) allowed by the model. ${ }^{10,15,16}$

Allowing for a changing population, and converting from continuous to discrete mathematics, creates the following model:

$$
\begin{gathered}
S_{t+1}=S_{t}+\left\lfloor\phi\left(S_{t}+I_{t}+E_{t}\right)\right\rceil-\left\lfloor\frac{\beta I_{t} S_{t}}{S_{t}+I_{t}+E_{t}}\right\rfloor+\left\lfloor\tau I_{t}\right\rceil-\left\lfloor\delta_{1} S_{t}\right\rceil \\
E_{t+1}=E_{t}+\left\lfloor\frac{\beta I_{t} S_{t}}{I_{t}+S_{t}+E_{t}}\right\rfloor-\left\lfloor\mu E_{t}\right\rceil-\left\lfloor\delta_{2} E_{t}\right\rceil \\
I_{t+1}=I_{t}+\left\lfloor\mu E_{t}\right\rceil-\left\lfloor\gamma I_{t}\right\rceil-\left\lfloor\tau I_{t}\right\rceil-\left\lfloor\delta_{3} I_{t}\right\rceil \\
R_{t+1}=\left\lfloor\gamma I_{t}\right\rceil+\left\lfloor\delta_{1} S_{t}\right\rceil+\left\lfloor\delta_{2} E_{t}\right\rceil+\left\lfloor\delta_{3} I_{t}\right\rceil
\end{gathered}
$$

The notation, $L \cdot 7$, represents a rounding function (formed from half of the ceiling function notation and half of the floor function notation) that forces the result to be a discrete value. ${ }^{15}$

The presented discrete model relies on a couple of core assumptions. First, the model relies on homogeneous mixing in which exactly one infective comes into contact with exactly one susceptible during each time step $(t, t+1)$. Therefore, it is only possible to spread the disease if the individual is actually infectious and not just infected (i.e., an individual in the exposed class cannot infect a susceptible). Second, the model assumes that the time it takes for an individual to transition from one subpopulation to another is negligible. Third, natural births and immigration results in only susceptible individuals, but individuals are able to emigrate or die from natural causes from any class. Therefore, $R$ represents individuals who have been removed from the population due emigration, natural deaths, or deaths as a result of the epidemic. Furthermore, at time $t=0, R_{0}=0$, but at time $t>0, R_{t}$ does not influence $S_{t+1}, I_{t+1}$, or $E_{t+1}$. Therefore, $R_{t}$ does not effect the interaction term given by:

$$
\left\lfloor\frac{\beta I_{t} S_{t}}{S_{t}+I_{t}+E_{t}}\right\rceil .
$$

Equation 14. 
Therefore the denominator for the interaction term is presented as $I_{t}+S_{t}+E_{t}$, rather than $N_{.}{ }^{3,6,10,15,16}$

In order to control the spread of the epidemic, one needs to impose a rate of vaccination to lower the transmission parameter, $B$. Letting $u$ represent the control vector and $u_{t}$ represent the rate of vaccination used during each time step $(t, t+1), B$ can be defined as $B=\beta(u)$. Therefore, $B$ is a known decreasing function in $u$. For binary control,

$$
\beta(u)=\left\{\begin{array}{l}
\beta(0)=B_{\max } \\
\beta(\sigma)=B_{\text {min }}
\end{array}\right.
$$

Equation 15.

where $B_{\max }>B_{\min }$. Since $B_{\max }$ and $B_{\min }$ are probabilities of transmission, both $B_{\max }, B_{\min } \in[0,1]$. For the purposes of the binary model presented, $u_{t} \in\{0, \sigma\}$, where one can either administer no vaccine (a zero vaccination rate) or administer the maximum vaccination rate, $\sigma$. Therefore, a relationship exists between whether a control is administered and the probability, $\beta$, of infection. If the rate of vaccination is zero, then the probability that the infection spreads should be at its highest, $B_{\max }$. If the rate of vaccination administered is at its maximum, then the probability that the infection spreads should be at its lowest, $B_{\text {min }}$. The latter increases to reflect that the cost of eradicating a disease increases as its prevalence decreases, since it is harder to control a small and decreasing fraction of infectives. ${ }^{3}$

There is a cost associated with administering the vaccine in order to control the current infectives, but there is also a cost associated with the cost per new infective. In the case of continuous mathematics, this can be modeled by minimizing the objective (loss) function

$$
L=\int_{0}^{T}\left[c_{1} s+c_{2} \gamma\right] e^{-\alpha t} d t
$$

Equation 16.

where $c_{1}$ is the social cost per infective, $c_{2}$ is the cost of control per unit level of program effort, $\alpha$ is a discount rate, and $T$ represents a finite time horizon. ${ }^{18}$

Since the approach presented in this paper relies on discrete mathematics, the loss function used to calculate the cost at a particular time step becomes:

$$
L_{t}(u)=\left\lfloor\frac{\beta I_{t} S_{t}}{S_{t}+I_{t}+E_{t}}\right\rceil C I+u_{t} \frac{S_{t}+I_{t}+E_{t}}{I_{t}+1},
$$

Equation 17.

where $C I$ is the cost per new infective and $u_{t}$ is the control vector value at the current time step. If the number of partitions associated with the possible rates of vaccination is changed, $u_{t}$ will update accordingly to allow for the change in granularity; thereby, influencing the cost associated with controlling the epidemic.

The cumulative loss function used to calculate the cumulative cost, $J_{t}$, is defined as follows:

$$
J_{t}= \begin{cases}0 & t=0 \\ J_{t-1}+L_{t} & t \geq 1 .\end{cases}
$$

Equation 18.

Therefore,

$$
J_{t+1}=J_{t}+\left\lfloor\frac{\beta\left(u_{t}\right) I_{t} S_{t}}{S_{t}+I_{t}+E_{t}}\right\rceil C I+u_{t} \frac{S_{t}+I_{t}+E_{t}}{I_{t}+1}
$$

Equation 19.

Therefore, the problem is to find the optimal control vector $u_{t}$ for $\{T \in \mathbb{N} \mid 0 \leq T<\infty\}$ that minimizes $J_{t} .{ }^{3,15}$

The problem can be expanded to allow for k-ary control in which the rate of vaccination does not need to be restricted to either no vaccination or full vaccination. Therefore $u_{t}=\left\{\frac{j}{k} \sigma, j=0, \ldots, k\right\} .{ }^{3}$ In order to apply partial rates of vaccination, two assumptions must be made: First, it is assumed that there is a linear association between the cost and the rate of vaccination being applied. Second, it is assumed that $B$ remains a decreasing function of $u$, where $B$ must be 
able to take on an infinite number of possible values rather than two points. ${ }^{3}$

Using the two intersection points, $\left(0, B_{\max }\right.$ and $\left(\sigma, B_{\min }\right)$, one can find the linear representation for $\beta$, as shown in Equation 20. ${ }^{15,16}$

$$
\beta(u)=\left(\frac{B_{\min }-B_{\max }}{\sigma}\right) u+B_{\max }
$$

Equation 20.

Since the algorithm that will be utilized to solve the minimization problem will rely on an "intelligent" search of the space of feasible solutions, a state space needs to be established. Let $T=(V, A)$ be a graph with the following node and arc set: $V:\left(I_{t}, J_{t}\right)$ and $A:\left(\left(I_{t}, J_{t}\right),\left(I_{t+1}, J_{t+1}\right)\right)$, such that:

$$
\begin{gathered}
I_{t+1}=I_{t}(u), u \in\{0, \sigma\} \\
J_{t+1}=J_{t}+L\left(I_{t}, u\right) .
\end{gathered}
$$

Equation 21.

Equation 22.

Figure 2 represents the initial time step in $T$ for a binary control tree. The root is defined by $I_{0}$ and $J_{0}$, where $I_{0}$ represents the initial number of infectives and $J_{0}=0$ since the initial cumulative cost is zero. The left child, denoted by $u=0$, represents a vaccination rate of zero. The right child, denoted by $u=\sigma$, represents applying the maximum rate of vaccination. The resulting nodes $\left(I_{1}^{0}, J_{1}^{0}\right)$ and $\left(I_{1}^{\sigma}, J_{1}^{\sigma}\right)$ represent the two children of the root, where the subscripts represent the current time step, $t=1$, and the the superscripts, 0 or a $\sigma$, represents whether the vaccination rate was zero or maximum. After the initial time step, the notation is simplified. $I_{i}$ is defined to be the number of infectives at

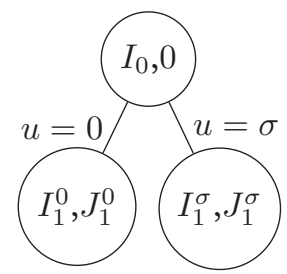

Figure 2. Initial Time Step of a Binary Control Tree.

node $i$ and $J_{i}$ represents the cumulative cost at node $i$ (i.e., the sum of all of the costs at each node on the path from the root to node $i$ ), and $t_{i}$ is the level of node $i$. This assumes that tree $T$ grows downward. ${ }^{15}$

To allow for additional rates of vaccination (partial levels of control), $u_{t}=\left\{\frac{j}{k} \sigma, j=0, \ldots, k\right\}$. Therefore, the resulting decision tree is formed by a root with $k+1$ children. Each child corresponds to a possible partial rate of vaccination, as shown in Figure 3. As in the case of the binary control decision tree, Figure 3 represents the initial time step in $T$, where the root is defined by $I_{0}$ and $J_{0}=0$. The root's $k+1$ children represents a zero rate of vaccination, maximum rate of vaccination, and $k-1$ rates of partial control. As before, each child node has a subscript denoting the current time step, and a superscript corresponding to the amount of control being applied. ${ }^{15}$

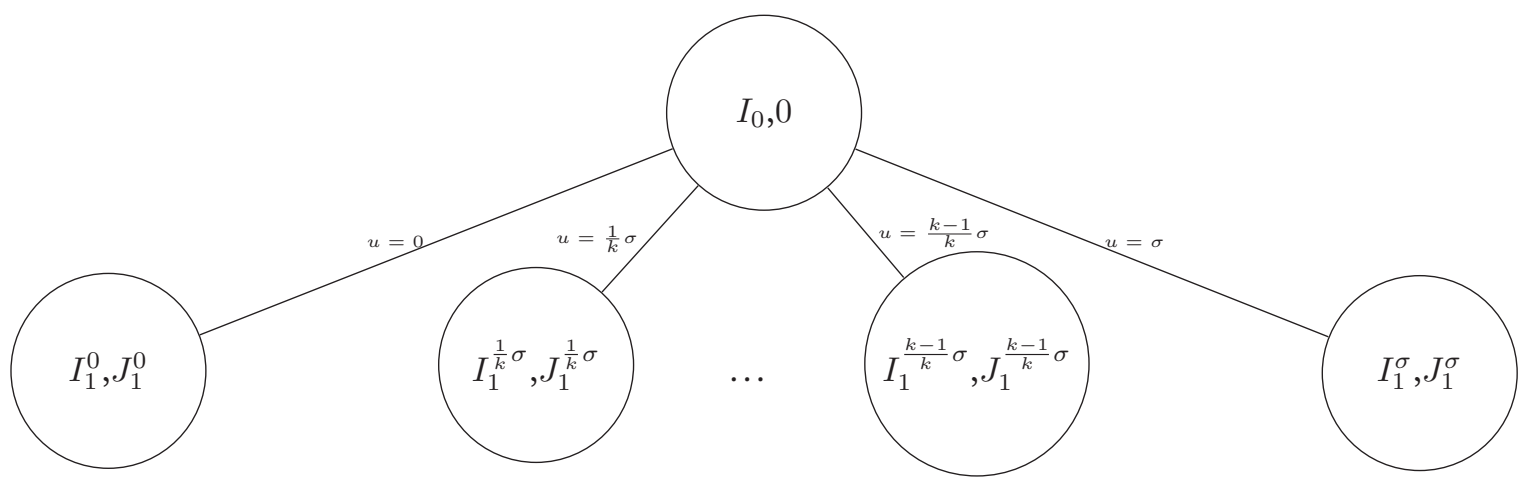

Figure 3. Initial Time Step of a K-ary Control Tree. 
In order to avoid an exponential expansion of the search space, especially as the number of partial vaccination rates goes up, a method of pruning subtrees should be utilized to eliminate paths that lead to leaves that are guaranteed not to yield optimal solutions.

Let $T$ be a state space tree. Let $i, j \in V(T)$ s.t. $i$ is a node at level $t_{i}$ and $j$ is a node at level $t_{j}, t_{i} \geq t_{j}, I_{i}=I_{j}$, and $J_{j} \leq J_{i}$. Then $T_{i}$, the subtree generated by node $i$, can be pruned. ${ }^{15}$

The algorithm utilized to find the optimal solution seeks to minimize the cumulative cost of controlling an epidemic over a finite time horizon. The algorithm begins with an initial number of infectives $I_{0}$ and an initial cumulative cost $J_{0}=0$. The loss function is calculated for each of the possible levels of vaccination. A comparison is made at each step according to the pruning technique above, so that large portions of the tree can be pruned. Pruning subtree $T_{i}$ results in a significant decrease in the number of paths that must be searched. Finally, the algorithm compares the resulting cumulative costs at the leaves of the remaining paths to find the optimal solution.

\section{NUMERICAL RESULTS}

To demonstrate the utility of the algorithm, a series of numerical results are presented. Unless otherwise specified, the parameter values are: $T=60, N_{0}=500, S_{0}=395, I_{0}=100, E_{0}=5, u_{t} \in[0,0.6], B_{\max }=0.75, B_{\min }=0.15, \tau=$ $0.13, \gamma=0.05, \sigma=0.6$, and $C I=0.65$. For each scenario, two discrete graphs are presented. The graph on the left represents the number of infected individuals (infectives) at each time step. The rate of vaccination graph on the right represents the optimal control solution which minimizes the cost of controlling the epidemic over the finite time horizon for the provided parameters.

The algorithm is initially applied to a binary control system, in which the rate of vaccination must be either 0 or 0.6 . Figure 4 shows the change in infected individuals and the applied rate of vaccination for a binary control system. Based on the provided parameters, the optimal solution to minimize the cost of controlling the epidemic is to consistently vaccinate at the maximum rate of 0.6 for the entire time horizon except for times $t=24, t=25, t=42$, and $t=$ 43. The initial vaccination effort is able to get the infectives down from 100 to 28 after the first 23 time steps. Once the vaccination effort is stopped, the number of infectives begins to increase very quickly to 51 individuals by $t=27$, therefore, requiring the vaccination efforts to resume. The same scenario occurs at $t=44$.

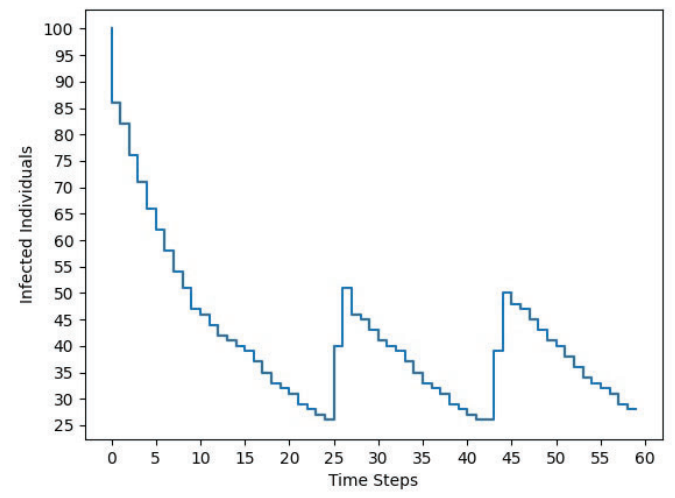

(a) Number of Infectives

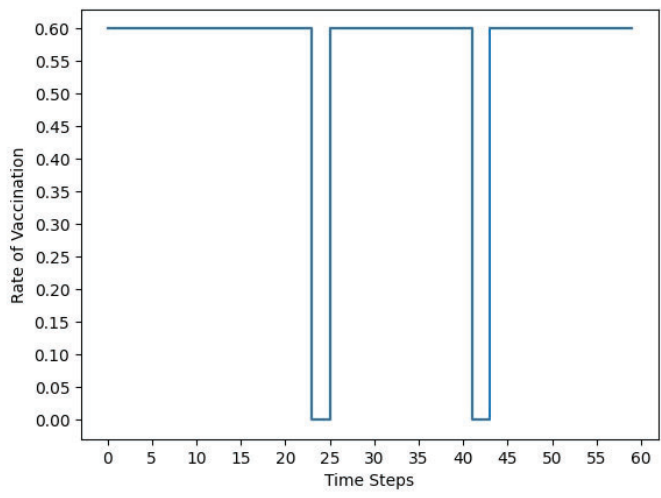

(b) Rate of Vaccination

Figure 4. Binary Control Experiment.

To demonstrate the algorithm's effectiveness for non-binary control scenarios, a series of k-ary control experiments are completed. Figures 5-7 demonstrates the effect of increasing the granularity of the vaccination efforts to allow for partial rates of vaccination to be implemented. Figure 5 demonstrates the effect of adding one additional rate of vaccina- 
tion. Therefore, instead of being restricted to either applying no vaccination or the maximum rate of vaccination, the 3 -ary control model also has the ability to choose to administer a $\frac{1}{2}$ vaccination rate. Since the maximum allowable rate of vaccination is $0.6, \mathrm{a} \frac{1}{2}$ vaccination rate would correspond to a rate of 0.3 . Hence, $u_{t}=\{0,0.3,0.6\}$. The optimal control solution shown is similar to the binary control. The algorithm administers the maximum rate of vaccination until time $t=23$ in order to rapidly decrease the number of infectives, after which an oscillatory pattern begins, toggling off and on the control as needed to maintain control of the epidemic, while minimizing the cost. At time $t=24$, $t=36$, and $t=52$, a 0.3 vaccination rate is implemented; therefore, the algorithm is taking advantage of the increase in granularity available. As far as the number of infectives goes versus time, the increase in granularity decreases the heights of each of the peaks and results in three peaks, rather than two as in the binary experiment.

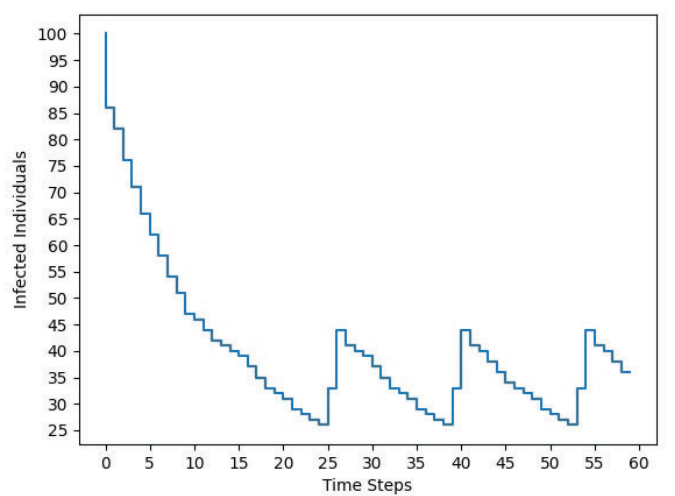

(a) Infectives

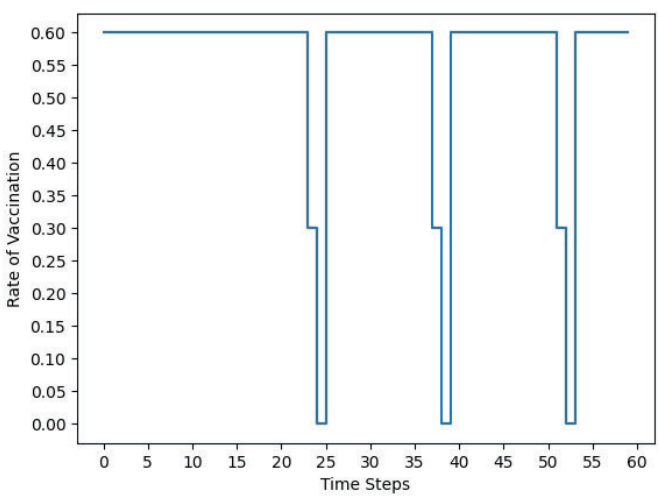

(b) Rate of Vaccination

Figure 5. 3-Ary Control Experiment.

Increasing the granularity to 10 levels of partial vaccination results in the outputs shown in Figure 6. As one can observe, the graph of the number of infectives begins to smooth out. There is only one significant peak, which occurs at time $t=26$ and some rippling between $t=35$ and $t=60$. The graph of the rate of vaccination demonstrates that the optimal solution is to control at the maximum rate for the first 22 time steps, before letting off to a vaccination rate of 0.47 at $t=23$ and 0.13 at $t=24$. Then, as the number of infectives increases, the need to apply a greater rate of vaccination occurs, therefore, the vaccination rate at $t=25, t=26$, and $t=27$ are $0.13,0.47$, and 0.6 , respectively. At this point, the rate of control applied oscilliates between 0.47 and 0.6 for the duration of the time horizon.

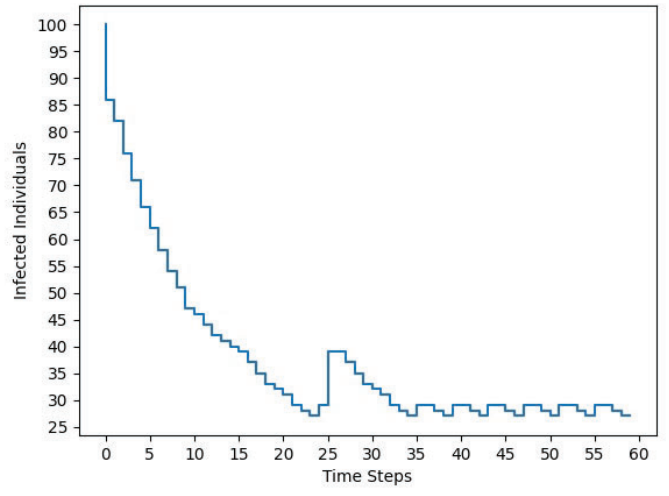

(a) Infectives

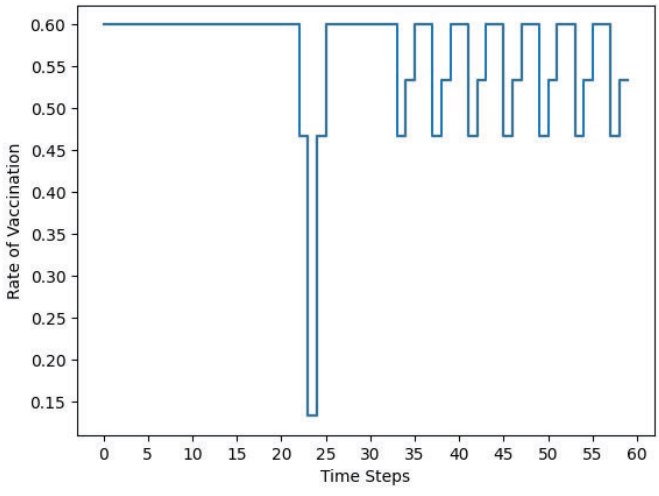

(b) Rate of Vaccination

Figure 6. 10-Ary Control Experiment. 
Finally, Figure 7 represents 20 levels of control. As expected, the graph of the infectives continues to smooth out and the rippling further disappears. This time the largest peak only gets back up to 35 infectives at time $t=26$. This is due to the increase in granularity as demonstrated in the rate of vaccination output. A similar oscillatory pattern occurs in the 20 -ary scenario as it did in the 10-ary scenario once the initial control of the epidemic occurs. Therefore, the rate of vaccination is stepped up only as needed to regain control of the small, rippling peaks.

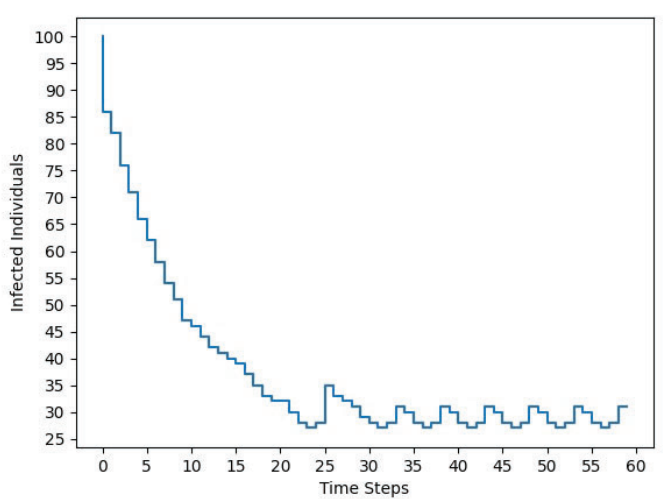

(a) Infectives

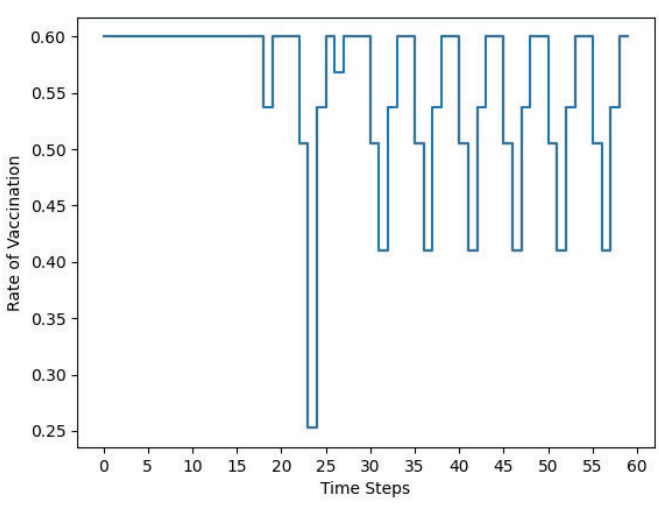

(b) Rate of Vaccination

Figure 7. 20-Ary Control Experiment.

Having explored the effects of increasing the granularity of the vaccination efforts, an investigation into the effects of changing the other parameters is carried out for the binary control experiment.

To begin, $\gamma$, the rate of death as a result of the infection is studied. Until this point, $\gamma$ was set to 0.05 . Figure 8 shows the effect of decreasing $\gamma$ to 0.02 , thereby making the disease less deadly. In this case, it becomes difficult to control the epidemic, since less individuals are leaving $I$ and entering $R$. Therefore, the number of infectives which can transmit the disease stays higher. Therefore, in order to get and maintain control of the spread of the epidemic, the optimal solution is to continuously vaccinate at the maximum rate. As one can observe from the graph of the infectives, it is necessary to control at the maximum rate to decrease the number of infectives from 100 to 49 , where it plateaus for the duration of the time horizon $(t=19$ through $t=60)$.

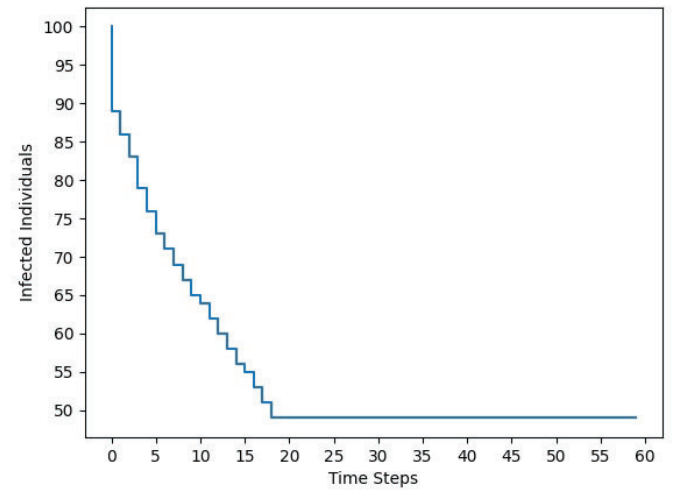

(a) Infectives

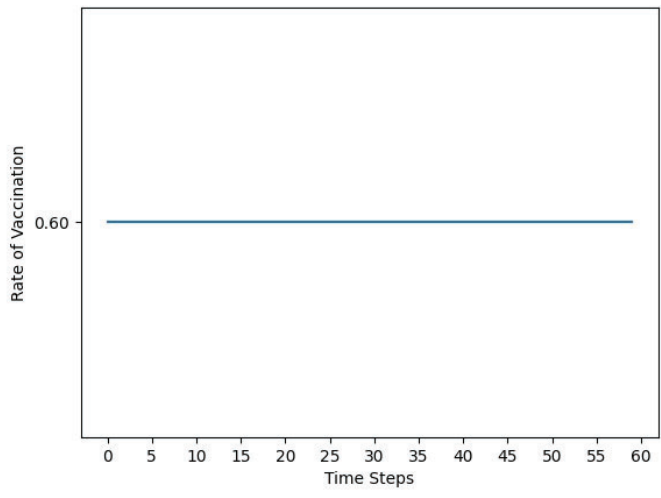

(b) Rate of Vaccination

Figure 8. Binary Control Experiment with $\gamma=0.02$. 
Figure 9 demonstrates the effect of increasing the deadliness of the disease to $\gamma=0.08$. In this case, less vaccination is required to control the epidemic, since the disease is decreasing the number of individuals in $I$ and moving them to $R$. Therefore, the disease's ability to kill its hosts acts as an internal control for the epidemic, so external intervention becomes less necessary that it did for $\gamma=0.05$. A general oscillatory pattern occurs with $\gamma=0.08$, but there are shorter periods in which the maximum rate of control is needed.

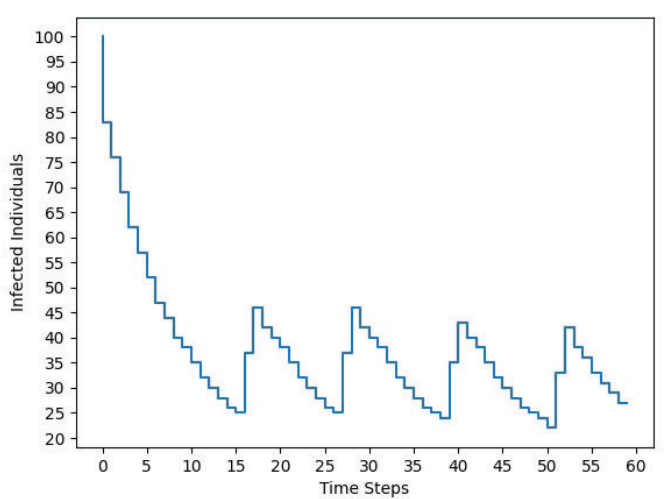

(a) Infectives

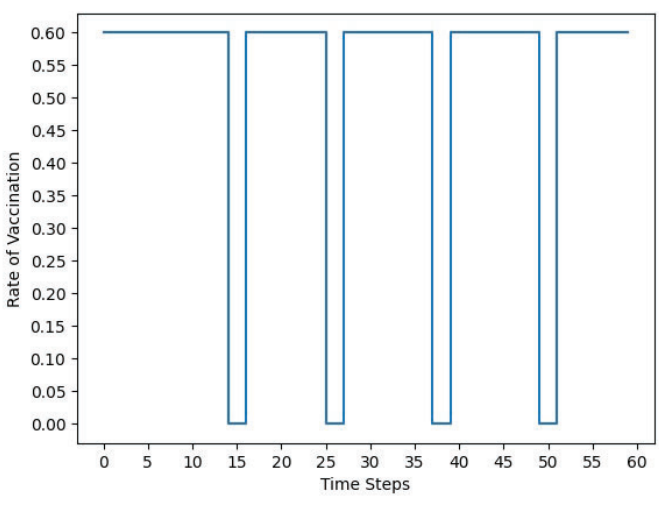

(b) Rate of Vaccination

Figure 9. Binary Control Experiment with $\gamma=0.08$.

Next, the effect of the recovery rate is analyzed. Until now the recovery rate, $\tau$, has been set to 0.13 . In Figures 10-11, the effects of altering the recovery rate are shown. Figure 10 represents the effect of decreasing $\tau$ from 0.13 to 0.08 . In this case, it becomes harder to control the epidemic, because individuals in the infective class are no longer recovering as quickly and reentering the class of susceptibles. Therefore, there are more infectives available at each time step able to transmit the infection to a susceptible. Therefore, it becomes necessary to continuously vaccinate at the maximum rate to maintain control of the epidemic and then maintain the constant number of infectives for the remaining portion of time horizon. There is a fairly consistent decrease (approximately linear in appearance) in the number of infectives between $t=1$ to $t=38$, after an immediate drop in the first time step. After time $t=38$, the number of infectives remains constant at 50 .

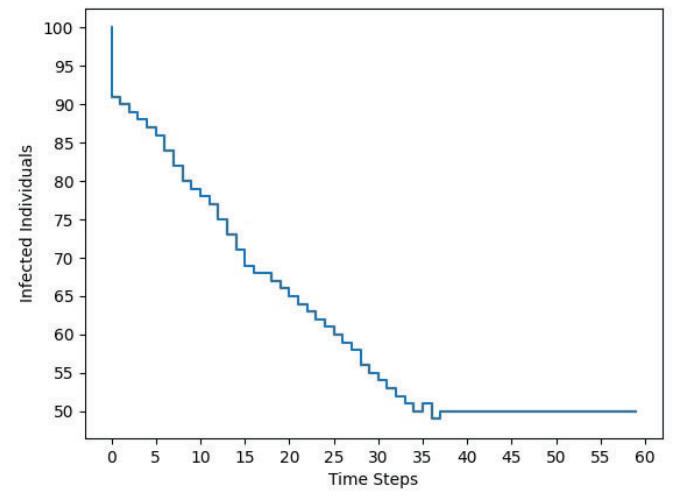

(a) Infectives

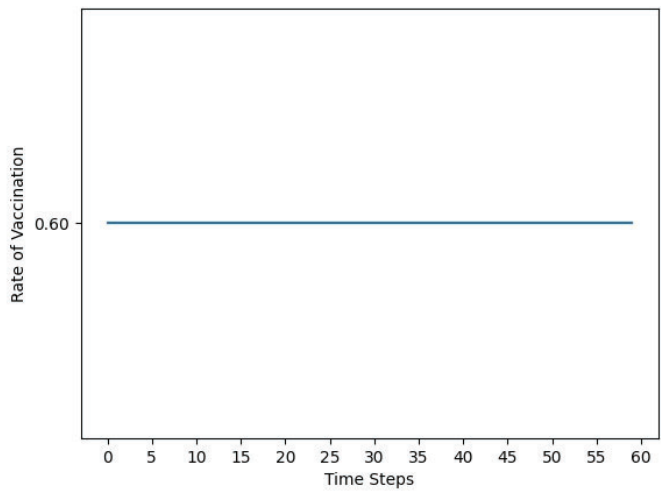

(b) Rate of Vaccination

Figure 10. Binary Control Experiment with $\tau=0.08$.

In Figure 11, $\tau$ is increased from 0.13 to 0.18 . In this case, the rate at recovery of infectious individuals is higher, so 
more individuals are moving between $I$ and $S$ in each time step. Therefore, the need to intervene with vaccination is decreased. The algorithm administers the maximum vaccination rate for the first 12 time steps to substantially decrease the number of initial infectives. After the initial vaccination efforts, it adopts an oscillatory pattern to maintain control with each peak in infectives.

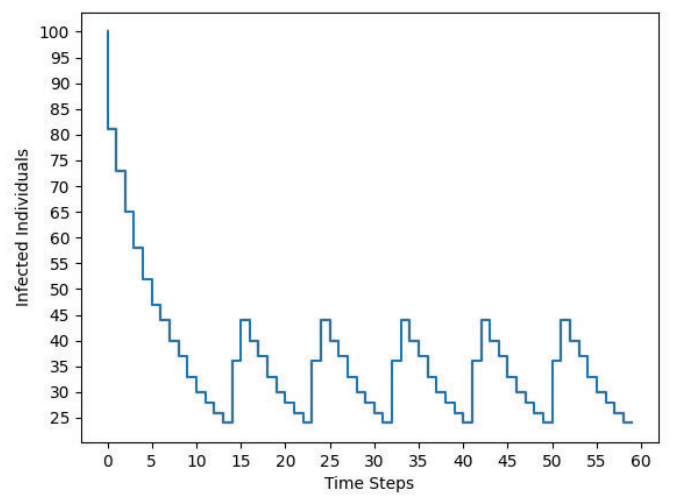

(a) Infectives

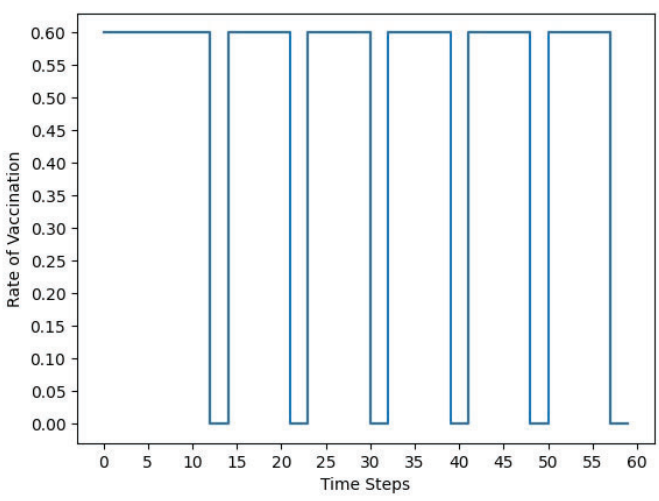

(b) Rate of Vaccination

Figure 11. Binary Control Experiment with $\tau=0.18$.

\section{CONCLUSIONS}

A discrete-oriented approach to epidemiological modeling and optimization is presented. A continuous model for an Susceptible/Exposed/Infective/Removed/Susceptible (SEIRS) model was converted to a discrete representation in order to more accurately reflect real-life discrete values. Additionally, the discrete model accounted for a changing population at each time step. The algorithm utilized sought to find the minimum cost necessary to control an epidemic under a finite time horizon and discrete state space. Using python coding software, the algorithm was effectively carried out under various parameters and the numerical results were presented.

There are a couple of notable results from the completion of the research and numerical simulations. First, increasing the granularity of the vaccination rates helped to smooth out the graph of infectives versus time, so there are smaller significant peaks in the number of infectives, and the size of any rippling in the number of infectives is also minimized. It is possible that a larger peak becomes smaller and the rippling that occurs afterwards may increase slightly, as the number of infectives may be spread out throughout the duration of the time horizon. This was observed in the graph of the infectives for the 10-ary versus 20-ary control experiments. A second notable result occurred with changing the values of $\tau$ or $\gamma$. If the rate of death for the disease $(\gamma)$ is decreased or the recovery rate $(\tau)$ is decreased, it became more difficult to control the epidemic. Therefore, the optimal solution was to administer the maximum rate of vaccination for the entire time horizon. If $\gamma$ or $\tau$ is increased, it becomes easier to control the epidemic. In the case of an increased $\tau$ value, the number of individuals recovering increased, so there were less infectives at each time step to spread the disease; therefore, less vaccination efforts were required to control the epidemic. In the case of an increased $\gamma$ value, the infection's increase in deadliness results in less need to vaccinate, since the disease kills more infectives at each time step; therefore, preventing the ability to transmit the disease to additional individuals.

\section{ACKNOWLEDGEMENTS}

The authors thank Madonna University's Department of Mathematics and Computer Science faculty and staff for their help and support. The authors also thank the reviewers of this work for their time and valuable feedback. 


\section{REFERENCES}

1. Althaus, C.L. and Heng, K. (2020) The approximately universal shapes of epidemic curves in the SusceptibleExposed-Infectious-Recovered (SEIR) model, Scientic Reports. https://doi.org/10.1038/s41598-020-76563-8

2. Arotaritei, D., Corciova, C., Constantin, G. (2018) Mathematical Models of Measles by Differential Equations in Virtual Education, The International Scientific Conference eLearning and Software for Education 3, 369374.https://doi.org/10.12753/2066-026X-18-197

3. Blount, S. and Galambosi, A. and Yakowitz, S. (1997) Nonlinear and Dynamic Programming for Epidemic Intervention, Applied Mathematics and Computation 86, 123-136. https://doi.org/10.1016/S0096-3003(96)00177-4

4. Brauer, F. (2009) Mathematical Epidemiology is not an Oxymoron, BMC Public Health 9. https://doi.org/10.1186/1471-2458-9-S1-S2

5. Cruz-pacheco, G., Esteva, L., Vargas, C. (2014) Vaccination Strategies for SIR Vector-Transmitted Diseases, BMB 76, 2073-2090. https://doi.org/10.1007/s11538-014-9999-6

6. Daley, D.J. and Gani, J. and Yakowitz, S. (2000) An Epidemic with Individual Infectivities and Susceptibilities, Mathematical and Computer Modeling 32, 155-157.

7. Fan X, Wang L, Teng Z. (2016) Global dynamics for a class of discrete SEIRS epidemic models with general nonlinear incidence, Advances in Difference Equations. https://doi.org/10.1186/s13662-016-0846-y

8. Gerberry, D.J., Milner, F.A. (2009) An SEIQR model for childhood diseases, JOMB 59, 535-561. https://doi.org/10.1007/s00285-008-0239-2

9. Greer, M.L, Palin, K.A. (2012) Students in Differential Equations and Epidemiology Model a Campus Outbreak of pH1N1, Journal of Microbiology and Biology Education 13, 183-185. http://dx.doi.org/10.1128/jmbe.v13.i2.429

10. Hethcote, H.W. (2000) The Mathematics of Infectious Diseases, SIAM Review 42, 599-653. https://doi.org/10.1137/S0036144500371907

11. Jarynowski, A. (2013) Human-Human Interaction: Epidemiology, arXiv. https://arxiv.org/ftp/arxiv/papers/1303/1303.7208.pdf

12. Jia, J., Xiao, J. (2018) Stability analysis of a disease resistance SEIRS model with nonlinear incidence rate. Adv Differ Equ 75. https://doi.org/10.1186/s13662-018-1494-1

13. Ma, J. (2020) Estimating epidemic exponential growth rate and basic reproduction number, Infectious Disease Modelling. https://doi.org/10.1016/j.idm.2019.12.009.

14. Mummert, A. (2013) Studying the recovery procedure for the time-dependent transmission rate(s) in epidemic models, JOMB 67, 483-507. https://wrww.doi.org/10.1007/s00285-012-0558-1

15. Nierman, R. (2017) Combinatorial Approaches to Continuous Problems, Oakland University. https://wrww.proquest.com/docview/1917681683

16. Nierman, R. and Kruk, S. (2017) An Optimal Intervention Experiment with Multiple Controls, AMO 19. https://camo.ici.ro/journal/vol19/v19a1.pdf

17. Salpeter, E. and Salpeter, S. (1998) Mathematical Model for the Epidemiology of Tuberculosis, with Estimates of the Reproductive Number and Infection-Delay Function, AJE 147, 398-406. https://doi.org/10.1093/oxfordjournals.aje.a009463

18. Sethi, Suresh P., (1974) Quantitative Guidelines For Communicable Disease Control Program: A Complete Synthesis, Biometrics 30, 681-691. https://doi.org/10.2307/2529232

\section{ABOUT THE STUDENT AUTHOR}

Tanner Snyder graduated from Madonna University in 2019 with a degree in Mathematics and a minor in Computer Science. He is currently working on his Masters Degree in Industrial Applied Mathematics at Oakland University with a focus in Discrete Mathematics. 


\section{PRESS SUMMARY}

This research presents an optimal control model for epidemiology assuming a finite time period and discrete time steps. This research develops a Susceptible/Exposed/Infective/Removed/Susceptible (SEIRS) model and incorporates a changing population variable to account for births/deaths and immigration/emigration. A series of numerical results are presented using python software and graph generation. The research presented is of particular interest in the wake of the COVID-19 pandemic and can be applied to various SEIRS epidemics for research and modeling purposes. 